\title{
An EM-like algorithm for semi- and non-parametric estimation in multivariate mixtures
}

\author{
Tatiana Benaglia ${ }^{1}$ Didier Chauveau ${ }^{2}$ David R. Hunter ${ }^{1,3}$ \\ August 2008 \\ ${ }^{1}$ Pennsylvania State University, USA \\ ${ }^{2}$ Université d'Orléans \& CNRS UMR 6628, France \\ ${ }^{3}$ Le Studium, CNRS Orléans, France \\ This research is partially supported by NSF Award SES-0518772.
}

\begin{abstract}
We propose an algorithm for nonparametric estimation for finite mixtures of multivariate random vectors that strongly resembles a true EM algorithm. The vectors are assumed to have independent coordinates conditional upon knowing which mixture component from which they come, but otherwise their density functions are completely unspecified. Sometimes, the density functions may be partially specified by Euclidean parameters, a case we call semiparametric. Our algorithm is much more flexible and easily applicable than existing algorithms in the literature; it can be extended to any number of mixture components and any number of vector coordinates of the multivariate observations. Thus it may be applied even in situations where the model is not identifiable, so care is called for when using it in situations for which identifiability is difficult to establish conclusively. Our algorithm yields much smaller mean integrated squared errors than an alternative algorithm in a simulation study. In another example using a real dataset, it provides new insights that extend previous analyses. Finally, we present two different variations of our algorithm, one stochastic and one deterministic, and find anecdotal evidence that there is not a great deal of difference between the performance of these two variants.
\end{abstract}

Keywords: EM algorithm, kernel density estimation, multivariate mixture, nonparametric mixture. 


\section{Introduction and motivating example}

Suppose the vectors $\mathbf{X}_{1}, \ldots, \mathbf{X}_{n}$ are a simple random sample from a finite

mixture of $m>1$ arbitrary distributions. The density of each $\mathbf{X}_{i}$ may be written

$$
g_{\varphi}\left(\mathbf{x}_{i}\right)=\sum_{j=1}^{m} \lambda_{j} \phi_{j}\left(\mathbf{x}_{i}\right),
$$

where $\mathbf{x}_{i} \in \mathbb{R}^{r}, \varphi^{t}=\left(\boldsymbol{\lambda}^{t}, \boldsymbol{\phi}^{t}\right)=\left(\lambda_{1}, \ldots, \lambda_{m}, \phi_{1}, \ldots, \phi_{m}\right)$ denotes the parameter, and the $\lambda_{j}$ are positive and sum to unity. We assume that the $\phi_{j}$ are drawn from some family $\mathcal{F}$ of multivariate density functions (say, absolutely continuous with respect to Lebesgue measure). Model (1) is not identifiable if no restrictions are placed on $\mathcal{F}$, where by "identifiable" we mean that $g_{\varphi}$ has a unique representation of the form (1) and we do not consider that "label-switching" - i.e., reordering the $m$ pairs $\left(\lambda_{1}, \phi_{1}\right), \ldots,\left(\lambda_{m}, \phi_{m}\right)-$ produces a distinct representation.

A common restriction placed on $\mathcal{F}$, which we adopt throughout this article, is that each joint density $\phi_{j}(\cdot)$ is equal to the product of its marginal densities. In other words, the coordinates of the $\mathbf{X}_{i}$ vector are independent, conditional on the subpopulation or component $\left(\phi_{1}\right.$ through $\left.\phi_{m}\right)$ from which $\mathbf{X}_{i}$ is drawn. Therefore, model (1) becomes

$$
g_{\boldsymbol{\varphi}}\left(\mathbf{x}_{i}\right)=\sum_{j=1}^{m} \lambda_{j} \prod_{k=1}^{r} f_{j k}\left(x_{i k}\right),
$$

where the function $f(\cdot)$, with or without subscripts, will always denote a univariate density function. If the $f_{j k}(\cdot)$ are assumed to come from a particular parametric family of densities, then standard univariate mixture model techniques (cf. MacLachlan and Peel, 2000 or Titterington et al., 1985) may easily be extended to the multivariate case. However, we wish to avoid the parametric assumption; in this article, we introduce an algorithm for esti- 
mating the parameter vector $\varphi$ in model (2), where we do not assume that $f_{j k}(\cdot)$ comes from a family of densities that may be indexed by a finitedimensional parameter vector.

Some authors (e.g., Hall and Zhou, 2003) consider model (2) in its full generality. Others (e.g., Hettmansperger and Thomas, 2000) consider the special case in which the density $f_{j k}(\cdot)$ does not depend on $k$ - that is, in which the $\mathbf{X}_{i}$ are not only conditionally independent but identically distributed as well:

$$
g_{\boldsymbol{\varphi}}\left(\mathbf{x}_{i}\right)=\sum_{j=1}^{m} \lambda_{j} \prod_{k=1}^{r} f_{j}\left(x_{i k}\right),
$$

What distinguishes model (2) from model (3) is the assumption in the latter that $f_{j 1}(\cdot)=\cdots=f_{j r}(\cdot)$ for all $j$. In some situations, this assumption may be too restrictive; yet we may not wish to employ the fully general model (2) because there is reason to assume that some of the $f_{j 1}(\cdot), \ldots, f_{j r}(\cdot)$ are the same. For instance, in the water-level dataset discussed later in this section, there are $r=8$ coordinates per observation, yet because of the experimental methodology used to collect the data, it is reasonable to assume that the eight coordinates may be organized into four blocks of two each, where the densities within each block are identical but we do not assume a priori that the four blocks share a common density function.

Thus, in order to encompass both the special case (3) and the more general case (2) simultaneously in this article, we introduce one further bit of notation: We will allow that the coordinates of $\mathbf{X}_{i}$ are conditionally independent and that there exist blocks of coordinates that are also identically distributed. These blocks may all be of size one so that case (2) is still covered, or there may exist only a single block of size $r$, which is case (3). If we let $b_{k}$ denote the block to which the $k$ th coordinate belongs, where $1 \leq b_{k} \leq B$ and $B$ is the total number of such blocks, then equation (2) is 
replaced by

$$
g_{\boldsymbol{\varphi}}\left(\mathbf{x}_{i}\right)=\sum_{j=1}^{m} \lambda_{j} \prod_{k=1}^{r} f_{j b_{k}}\left(x_{i k}\right) .
$$

With so many different subscripts, the notation itself can become an impediment to understanding. Thus, we will remain consistent in our use of notation and terminology throughout the article. In particular, we use the terms component and coordinate only to refer, respectively, to one of the distributions (subpopulations) making up the mixture and one of the repeated measurements making up an observation. The indices $i, j, k$, and $\ell$ will always denote a generic individual, component, coordinate, and block, respectively. Therefore, we will always have $1 \leq i \leq n, 1 \leq j \leq m, 1 \leq k \leq r$, and $1 \leq \ell \leq B$. (Also note that $m, r$, and $B$ stand for mixture components, repeated measurements, and blocks, and as usual $n$ is the sample size.)

To further elucidate model (4), consider as an example an experiment involving 405 children aged 11 to 16 years subjected to a water-level task as described by Thomas et al. (1993). Each child is presented with eight rectangular vessels on a sheet of paper, each tilted to one of $r=8$ clock-hour orientations: in order of presentation to the subjects, these orientations are 11, 4, 2, 7, 10, 5, 1, and 8 o'clock. Each vessel was on a separate sheet of paper and appeared much like the small reproductions in the plots of Figure 1 (see Thomas et al., 1993, p. 40). The children's task was to draw a line representing the surface of still liquid in the closed, tilted vessel in each picture. Each such line describes two points of intersection with the sides of the vessel; the acute angle, in degrees, formed between the horizontal and the line passing through these two points was measured for each response. The sign of each such measurement was taken to be the sign of the slope of the line.

This water-level dataset (with $r=8$ and $m=3$ or 4 ) and our analysis of 

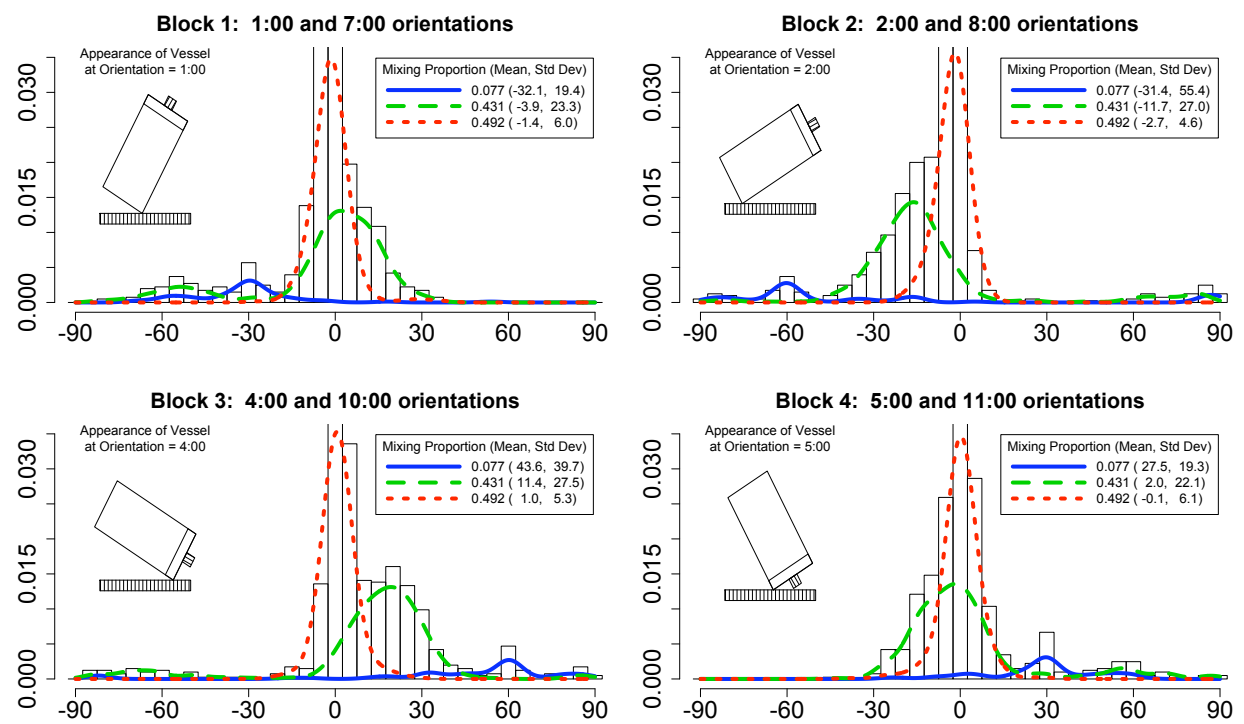

Figure 1: The water-level data are analyzed using our algorithm, assuming model (4) with three mixture components $(m=3)$ and four coordinate blocks $(B=4)$ in which opposite clock-face orientations are assumed to lead to conditionally independent and identically distributed responses. The means and standard deviations are for interpretation only; they are not part of the model, which is fully nonparametric except for the mixing proportions $\lambda_{j}$.

it will be described in further detail in Section 5.3; for now, we state only that we know of no other algorithm currently capable of producing similar results. The method of Hall et al. (2005) could potentially be extended to this case, but this method appears to be extremely complicated computationally for $m>2$ or $r>3$. The method of Qin and Leung (2006) can be used in this case, but this method entails more restrictive assumptions than those of model (2), namely, that the log-ratios of all component densities (for a given coordinate) are quadatic, or more generally parametric, functions of the observations. Some other methods have been proposed that extend easily to the case of $m>2$ or $r>3$ - indeed, the same water-level dataset 
has been analyzed by other authors (Hettmansperger and Thomas, 2000; Elmore et al., 2004) - yet these methods rely on the assumption that the $r=8$ coordinates are all identically distributed. Our method appears to be the only one that is both fully general and easily extendible to any values $m$ and $r$ for which model (2) is identifiable.

In Section 2 of this article, we offer a fuller discussion of previous work in this area, including the vexing issue of identifiability. We introduce our algorithm in Section 3 and describe several modifications of the basic algorithm and model in Section 4. Section 5 is devoted to empirical study of the algorithm, both through simulation studies and analysis of a real dataset. Whenever possible in Section 5, we compare our results with those reported in the literature for other known methods.

\section{Identifiability and previous work}

An interesting question is how restrictive the assumptions on $f_{j b_{k}}(\cdot)$ must be in order to ensure that model (4) is identifiable. For instance, in the univariate $(r=1)$ case, Bordes et al. (2006) and Hunter et al. (2007) found that when $f_{j}(x)=f\left(x-\mu_{j}\right)$ for some density $f(\cdot)$ that is symmetric about zero, the mixture density $g_{\varphi}(x)$ admits a unique representation whenever $m \leq 3$, except in certain special cases that are easily enumerable.

In the multivariate case, Hall and Zhou (2003) showed that for two components $(m=2)$, the most general model (2) — the same as model (4) with $b_{k}=k$ for all $k$ - is identifiable as long as $r \geq 3$ under an additional assumption that Hall and Zhou (2003) term "irreducibility" — namely, that none of the bivariate marginals of the mixture density factors into the product of its univariate marginals. Hall et al. (2005) extended these results, suggesting that model (2) is a case in which the conditions on $m$ necessary 
for identifiability get less restrictive as the dimension $r$ increases; or, as they themselves put it, this is a case in which "from at least one point of view, the 'curse of dimensionality' works in reverse."

We use the term "nonparametric" to describe the case in which no assumptions are made about the form of the $f_{j b_{k}}(\cdot)$, even though the parameter $\boldsymbol{\lambda}$ is of course Euclidean. We reserve the term "semiparametric" for the case in which $f_{j b_{k}}(\cdot)$ is partly specified by a finite-valued parameter, such as the case discussed above in which $f_{j}(x)=f\left(x-\mu_{j}\right)$ for a symmetric but otherwise completely unspecified density $f(\cdot)$. Note that Lindsay (1995) speaks of "nonparametric mixture modeling" in a different sense: The family $\mathcal{F}$ from which the component densities come is fully specified up to a parameter $\boldsymbol{\theta}$, but the mixing distribution from which the $\boldsymbol{\theta}$ are drawn, rather than having finite support of known cardinality $m$ as in the present article, is assumed to be completely unspecified a priori.

When $b_{k}=k$ for all $k$, several authors have recently addressed the problem of estimating the $f_{j b_{k}}$ in model (4). Yet the estimation methods they propose appear to apply in only limited cases or to require additional assumptions. Qin and Leung (2006) and Leung and Qin (2006) adapt the exponential tilt model of Anderson (1979) and apply their methods to the cases when $m=2$ and $r=2$ or $r=3$. While these methods do appear to be extendible beyond these cases, they always require an additional assumption, which we describe in Equation (21) in Section 5.2. Hall et al. (2005) give estimators based on inversion of mixture models that apply only in the case when $m=2$ and $r=3$; analytical difficulties appear to hinder the application of their method beyond this case. Even in the case $r=1$, where restrictions as described at the beginning of Section 2 must be placed on $f_{j}(\cdot)$ in order to ensure identifiability, the estimation methods of Bordes et 
al. (2006) and Hunter et al. (2007) are difficult if not impossible to apply beyond the case $m=2$. We discuss this case in Section 4.3 and use it as the basis for the numerical example of Section 5.4.

By contrast, in the case of continuous data when $b_{k}=1$ for all $k$ - that is, the case of conditionally independent and identically distributed coordinates - several other authors (Hettmansperger and Thomas, 2000; CruzMedina et al., 2004; Elmore et al., 2004) have developed a different estimation method. This method, the cutpoint approach, discretizes the continuous measurements by replacing each observation $\left(x_{i 1}, \ldots, x_{i r}\right)$ by a multinomial vector $\left(n_{1}, \ldots, n_{p}\right)$, where

$$
n_{a}=\sum_{k=1}^{r} I\left\{c_{a-1}<x_{i k} \leq c_{a}\right\}, \quad 1 \leq a \leq p,
$$

and the cutpoints $-\infty=c_{0}<c_{1}<\cdots<c_{p}=\infty$ are specified by the experimenter. The cutpoint approach is completely general in the sense that it can be applied to any number of components $m$ and any number of repeated measures $r$, just as long as $r \geq 2 m-1$, a condition that guarantees identifiability (Elmore and Wang, 2003). However, some information is lost in the discretization step and for this reason it becomes difficult to easily obtain density estimates of the component densities. Furthermore, even if the assumption of conditional independence is warranted, the extra assumption of identically distributed coordinates may not be; and the cutpoint method collapses when the coordinates are not identically distributed.

Here, we take a different approach and adapt an algorithm of Bordes et al (2007), which is presented by those authors as a stochastic algorithm for the particular univariate case of model (1) under the assumption that $\phi_{j}(x)=$ $f\left(x-\mu_{j}\right)$ for some symmetric density $f(x)$. We demonstrate how to extend the algorithm to model (4) and eliminate the stochasticity. Our algorithm 
combines the best features of all the algorithms discussed previously: It is simple to program, it is applicable to any $m$ and $r$ as well as any set of blocks $b_{k}$, and it gives kernel-density-like estimates for each of the $f_{j b_{k}}$.

Yet with such flexibility also comes a bit of danger, since the identifiability question for the general model (4) has not yet been settled. Hall et al. (2005) discuss this question and give a lower bound on $r$, as a function of $m$, that is necessary in order to guarantee identifiability: They state that $r$ and $m$ should satisfy $2^{r}-1 \geq m r+1$. Yet they do not give an explicit bound that is sufficient to guarantee identifiability; however, Elmore et al. (2005) prove that such a (finite) lower bound exists.

Since extending our estimation method to an arbitrary number of coordinates or mixture components is very easy - unlike any previously published algorithms for this problem - we are in a position in which practice is more advanced than theory. Thus, it is prudent to exercise caution when trying to fit a model for which the identifiability question is not settled. The water-level data of Section 1 provide such an example if we take $m=3$ or $m=4$. We discuss this example in more detail, and give reasons that we are fairly confident about interpreting our results, in Section 5.3. We also return briefly to the question of the behavior of our method for nonidentifiable models in Section 6.

\section{$3 \quad$ Estimating the parameters}

We propose both a refinement and a generalization of the algorithm of Bordes et al. (2007). Although we use the term EM in connection with this algorithm, we stress that this algorithm is not an EM algorithm in the usual sense (Dempster et al., 1977) because there is no likelihood that this algorithm may be shown to maximize. However, we retain the name "EM" 
because the algorithm strongly resembles a true EM algorithm for the parametric mixture case, i.e., the case in which $\mathcal{F}$ is a family indexed by some Euclidean parameter. For instance, as in an EM algorithm for mixtures, we define $Z_{i j} \in\{0,1\}$ to be a Bernoulli random variable indicating that individual $i$ comes from component $j$. Since each individual comes from exactly one component, this implies $\sum_{j=1}^{m} Z_{i j}=1$. Thus, the complete data is the set of all $\left(\mathbf{x}_{i}, \mathbf{Z}_{i}\right), 1 \leq i \leq n$.

\subsection{The nonparametric EM algorithm}

The algorithm described here is implemented in an $\mathrm{R}$ package ( $\mathrm{R}$ Development Core Team, 2008) called mixtools (Young et al., 2008), available online from the Comprehensive R Archive Network (CRAN) and also at the JCGS website. Suppose we are given initial values $\varphi^{0}=\left(\lambda^{0}, \mathbf{f}^{0}\right)$. Then for $t=1,2, \ldots$, we follow these three steps:

1. E-step: Calculate the "posterior" probabilities (conditional on the data and $\varphi^{t}$ ) of component inclusion,

$$
\begin{aligned}
p_{i j}^{t} & \stackrel{\text { def }}{=} P_{\boldsymbol{\varphi}^{t}}\left(Z_{i j}=1 \mid \mathbf{x}_{i}\right) \\
& =\frac{\lambda_{j}^{t} \prod_{k=1}^{r} f_{j b_{k}}^{t}\left(x_{i k}\right)}{\sum_{j^{\prime}=1}^{m} \lambda_{j^{\prime}}^{t} \prod_{k=1}^{r} f_{j^{\prime} b_{k}}^{t}\left(x_{i k}\right)}
\end{aligned}
$$

for all $i=1, \ldots, n$ and $j=1, \ldots, m$.

2. M-step: Set

$$
\lambda_{j}^{t+1}=\frac{1}{n} \sum_{i=1}^{n} p_{i j}^{t}
$$

for $j=1, \ldots, m$. 
3. Nonparametric density estimation step: For any real $u$, define for each component $j \in\{1, \ldots, m\}$ and each block $\ell \in\{1, \ldots, B\}$

$$
\begin{aligned}
f_{j \ell}^{t+1}(u) & =\frac{\frac{1}{h} \sum_{k=1}^{r} \sum_{i=1}^{n} p_{i j}^{t} I\left\{b_{k}=\ell\right\} K\left(\frac{u-x_{i k}}{h}\right)}{\sum_{k=1}^{r} \sum_{i=1}^{n} p_{i j}^{t} I\left\{b_{k}=\ell\right\}} \\
& =\frac{1}{n h C_{\ell} \lambda_{j}^{t+1}} \sum_{k=1}^{r} \sum_{i=1}^{n} p_{i j}^{t} I\left\{b_{k}=\ell\right\} K\left(\frac{u-x_{i k}}{h}\right),
\end{aligned}
$$

where $K(\cdot)$ is a kernel density function, $h$ is a bandwidth chosen by the user, and

$$
C_{\ell}=\sum_{k=1}^{r} I\left\{b_{k}=\ell\right\}
$$

is the number of coordinates in the $\ell$ th block. Note that in the case in which $b_{k}=k$ for all $k$, equation (8) becomes

$$
f_{j k}^{t+1}(u)=\frac{1}{n h \lambda_{j}^{t+1}} \sum_{i=1}^{n} p_{i j}^{t} K\left(\frac{u-x_{i k}}{h}\right) .
$$

In the original Bordes et al (2007) algorithm, the nonparametric density estimation step differs in that $p_{i j}^{t}$ is replaced by $z_{i j}^{*}$ in equation (8), where $\left(z_{i 1}^{*}, \ldots, z_{i m}^{*}\right)$ is a simulated multinomial random variable with a single trial and with probability vector given by $\left(p_{i 1}^{t}, \ldots, p_{i m}^{t}\right)$. Thus, the original algorithm has a stochastic element. In various tests, we find consistent empirical evidence that the deterministic version presented here is slightly, though not overwhelmingly, more efficient than the stochastic version. An example of such a comparison is given in section 5.4. Because the deterministic algorithm does not require any additional overhead relative to the stochastic algorithm, we use it here exclusively.

To initialize the algorithm, it is often easier to start with an initial $n \times m$ matrix $\mathbf{P}^{0}=\left(p_{i j}^{0}\right)$ than with an initial parameter vector $\varphi^{0}$. Thus, during the first iteration, we skip directly to the M-step. To obtain this $\mathbf{P}^{0}$ matrix, 
it is possible to use (say) a k-means clustering algorithm to assign each observation to one of the components. This procedure forces $\mathbf{P}^{0}$ to consist of just zeros and ones, but we find that it works well in practice.

\subsection{Bandwidth and kernel selection}

The density estimation step in the algorithm above relies on a kernel density $K(\cdot)$ and a bandwidth $h$. Kernel density estimation is a well-studied topic in statistics, and for our implementation in the mixtools package, we tried to adopt standard techniques. In particular, because much literature on this topic suggests that the choice of a kernel function does not have a dramatic impact on the resulting density estimate, we simply take $K(\cdot)$ to be the standard normal density function.

Choosing a bandwidth is a more complicated issue, since this choice affects the density estimates dramatically. Although we do not attempt a thorough exploration of this topic in the current article, we describe here some of our experience in choosing a bandwidth.

As a default value for the bandwidth $h$, we simply take the entire $n \times r$ dataset, treat it as a vector of length $n r$, and use the default bandwidth selection of the density function in $\mathrm{R}$ - namely, a rule of thumb due to Silverman (1986, page 48) in which

$$
h=0.9(n r)^{-1 / 5} \min \left\{\mathrm{SD}, \frac{\mathrm{IQR}}{1.34}\right\},
$$

where SD and IQR are the standard deviation and interquartile range of all $n r$ data values. This is a very crude method in the nonparametric mixture setting, and there are several reasons why it might produce an under- or overestimate. First, pooling all of the data implicitly treats all of the different components as though they are from the same distribution. This can lead to an inflation of the bandwidth, particularly if the mixture components' 
centers are well-separated, because in that case, the variability of the pooled dataset will be larger than that of the individual components. Similarly, if the vector coordinates are not identically distributed within each component, the bandwidth could be biased upward for the same reason.

Yet operating in the opposite direction is the fact that the expression $n r$ in equation (10) is an overestimate of the "true" sample size. This is especially true when each $b_{k}$ equals $k$ - where each of the $r$ coordinates gets a separate set of density estimates - in which case it may be sensible to eliminate the $r$ from the equation (10) entirely. But regardless of the values of $b_{k}$, there is also the fact that the "true" sample size from each component is actually some fraction of $n$, namely, about $\lambda_{j} n$ for the $j$ th component.

The arguments above show first of all that it would be useful to know something about the mixture structure in order to select a bandwidth. This suggests an iterative procedure in which the value of $h$ is modified, and the algorithm reapplied, after the output from the algorithm is obtained. Secondly, there is no reason that the bandwidth should be the same for each component or even for each block: It is easy to modify equation (8) by replacing $h$ by $h_{j}$ or $h_{j \ell}$.

A thorough exploration of the bandwidth question is therefore a research topic unto itself, so in the interest of simplicity we opt for the default value (10) in the simulation studies of Sections 5.1 and 5.2. For the water-level data discussed in Sections 1 and 5.3, where the visual appearance of the density estimates is important for a qualitative appreciation of the results, we find that the default value of $h=1.47$ produces a "bumpy-looking" set of density estimates, so we use a larger value that gives smoother results, namely $h=4$. We provide the very simple code for this example in Section 
5.3, and we encourage interested readers to test this example using the default bandwidth. We also find in a couple other datasets that the default (10) gives somewhat "bumpy-looking" results, suggesting that the default value tends to be smaller than a more optimal choice would be in general; yet our evidence for this is only anecdotal at this point.

\section{Modifications to the model and algorithm}

The general model of equation (4) and the algorithm of Section 3.1 may be modified in various ways. For example, the density-estimation bandwidth may be allowed to change for each component, each coordinate, or both, as mentioned in Section 3.2. We discuss some of these modifications here.

\subsection{Location-scale model}

There are some plausible models that are more restrictive than (4) but not as restrictive as the case in which all coordinates are identically distributed. For instance, if in equation (4) we write $\ell=b_{k}$ and suppose that

$$
f_{j \ell}(x)=\frac{1}{\sigma_{j \ell}} f_{j}\left(\frac{x-\mu_{j \ell}}{\sigma_{j \ell}}\right)
$$

for unknown parameters $\left(\boldsymbol{\mu}_{j}, \boldsymbol{\sigma}_{j}, f_{j}\right), j=1, \ldots, m$, then the totally nonparametric specification of $f_{j \ell}$ becomes a semiparametric specification (note that $\boldsymbol{\mu}_{j}$ and $\boldsymbol{\sigma}_{j}$ are both $B$-vectors). Though $f_{j}$ is fully unspecified, if we are willing to assume that it at least has a second moment then we may treat $\mu_{j l}$ and $\sigma_{j l}$ as mean and standard deviation parameters even though this is not implied by equation (11), an assumption that yields Equations (13) and (14) below. To implement the semiparametric EM algorithm in this case, equations (5) and (7) remain unchanged but it is necessary to modify equation (8) to account for the fact that all of the coordinates provide in- 
formation about the form of each $f_{j}$. Thus, in the case (11), equation (8) is replaced by

$$
f_{j}^{t+1}(u)=\frac{1}{n r h \lambda_{j}^{t+1}} \sum_{i=1}^{n} \sum_{k=1}^{r} p_{i j}^{t} K\left(\frac{u-x_{i k}+\mu_{j b_{k}}}{h \sigma_{j b_{k}}}\right) .
$$

Furthermore, the M-step also includes updates of the $\mu_{j \ell}$ and $\sigma_{j \ell}$ parameters for each $1 \leq j \leq m$ and $1 \leq \ell \leq B$ :

$$
\begin{array}{r}
\mu_{j \ell}^{t+1}=\frac{\sum_{i=1}^{n} \sum_{k=1}^{r} p_{i j}^{t} I\left\{b_{k}=\ell\right\} x_{i k}}{\sum_{i=1}^{n} \sum_{k=1}^{r} p_{i j}^{t} I\left\{b_{k}=\ell\right\}}=\frac{\sum_{i=1}^{n} \sum_{k=1}^{r} p_{i j}^{t} I\left\{b_{k}=\ell\right\} x_{i k}}{n \lambda_{j}^{t+1} C_{\ell}} \\
\sigma_{j \ell}^{t+1}=\left[\frac{1}{n C_{\ell} \lambda_{j}^{t+1}} \sum_{i=1}^{n} \sum_{k=1}^{r} p_{i j}^{t} I\left\{b_{k}=\ell\right\}\left(x_{i k}-\mu_{j \ell}^{t+1}\right)^{2}\right]^{1 / 2}
\end{array}
$$

Naturally, it is possible to place constraints on the $\boldsymbol{\mu}_{j}$ or $\boldsymbol{\sigma}_{j}$ vectors when this is sensible. For instance, if the mixture is purely a location mixture, then we might stipulate that $\boldsymbol{\sigma}_{j}=\boldsymbol{\sigma}$ for each $j$ and for some $B$-vector $\boldsymbol{\sigma}$. Similarly, we might stipulate that $\boldsymbol{\mu}_{j}=\boldsymbol{\mu}$ if the mixture is purely a scale mixture. In these latter two cases, note that we still allow the different blocks to have different scale and location parameters, though of course this may be restricted as well.

\subsection{Location-scale model, revisited}

We may obtain yet a different model by writing

$$
f_{j \ell}(x)=\frac{1}{\sigma_{j \ell}} f_{\ell}\left(\frac{x-\mu_{j \ell}}{\sigma_{j \ell}}\right)
$$

instead of equation (11). These two equations, which differ only in the replacement of a single $j$ by an $\ell$, in fact involve assumptions that are quite distinct. In equation (11), we are assuming that the coordinates within an 
individual have the same shape of distribution (depending on the individual's mixture component) but may differ by a location and scale factor; in equation (15), we are assuming that individual differences, i.e., the mixture components, only account for differences up to a location and scale parameter, but otherwise the distributions of different blocks of coordinates do not relate to one another in any way. Note also that the corresponding form of equation (8) looks quite different than its earlier counterpart in equation (12):

$$
f_{\ell}^{t+1}(u)=\frac{1}{n h C_{\ell}} \sum_{i=1}^{n} \sum_{j=1}^{m} \sum_{k=1}^{r} I\left\{b_{k}=\ell\right\} p_{i j}^{t} K\left(\frac{u-x_{i k}+\mu_{j \ell}}{h \sigma_{j \ell}}\right) .
$$

As a special case of both (11) and (15), we may assume that all coordinates in all components have the same distributional shape, summarized by the density $f(\cdot)$, and

$$
f_{j \ell}(x)=\frac{1}{\sigma_{j \ell}} f\left(\frac{x-\mu_{j \ell}}{\sigma_{j \ell}}\right) .
$$

In case (17), equation (8) becomes

$$
f^{t+1}(u)=\frac{1}{n r h} \sum_{i=1}^{n} \sum_{j=1}^{m} \sum_{k=1}^{r} p_{i j}^{t} K\left(\frac{u-x_{i k}+\mu_{j b_{k}}}{h \sigma_{j b_{k}}}\right) .
$$

\subsection{Symmetric components}

If we consider case (17) without repeated measures $(r=1)$ and for purely a location mixture $\left(\sigma_{j}=1, j=1, \ldots, m\right)$, then the model becomes

$$
g_{\varphi}\left(x_{i}\right)=\sum_{j=1}^{m} \lambda_{j} f\left(x_{i}-\mu_{j}\right)
$$

When $m=2$, equation (19) is exactly the location-shifted semiparametric mixture model that is proved identifiable by Bordes et al. (2006) and Hunter et al. (2007) under the additional assumptions that $\lambda_{1} \neq 1 / 2$ and that the 
density $f$ is symmetric about zero. This special case is also the model for which the original (semiparametric) stochastic EM algorithm is proposed in Bordes et al. (2007). In the non-stochastic version, equation (18) may be combined with a symmetrization step to give

$$
f^{t+1}(u)=\sum_{i=1}^{n} \sum_{j=1}^{m} \frac{p_{i j}^{t}}{2 n h}\left[K\left(\frac{u-x_{i}+\mu_{j}}{h}\right)+K\left(\frac{-u-x_{i}+\mu_{j}}{h}\right)\right] .
$$

A comparison of the stochastic and non-stochastic versions of this algorithm is given in section 5.4 .

\subsection{Changing block structure}

In Figure 1 summarizing the water-level results for three components (a dataset that is discussed further in Section 5.3), we see that the largest component, into which roughly half of the subjects fall, appears to have roughly the same density for all four blocks. We might therefore guess that for individuals in this component, observations $\mathbf{x}_{i}$ consist of 8 independent and identically distributed (i.i.d.) coordinates. Yet the remaining two components' observations do not appear to be identically distributed; the block structure exhibited in the plots, in which the eight coordinates fall into 4 blocks of two i.i.d. observations each, seems appropriate. It is therefore reasonable to allow the model to encompass the possibility that the block structure could be different in each component. In other words, equation (4) would be modified slightly to produce

$$
g_{\boldsymbol{\varphi}}\left(\mathbf{x}_{i}\right)=\sum_{j=1}^{m} \lambda_{j} \prod_{k=1}^{r} f_{j b_{j k}}\left(x_{i k}\right)
$$

in which the only difference is that $b_{k}$ has been replaced by $b_{j k}$ - thus, the block in which the $k$ th observation falls depends on $j$ as well.

Though this generalization of the model is not currently implemented in the mixtools package, it would be conceptually easy to do so. However, 
there is a theoretical issue that must be addressed in this case: Labelswitching becomes a problem. By "label-switching", we mean the result of permuting the labels of the $m$ components. When each component is assumed to follow the same model, it is not important which is labeled component 1 , which is labeled component 2 , etc. But if we now assume that component 1 (say) has i.i.d. coordinates whereas components 2 and 3 have a different block structure, then it is necessary to ensure that "component 1" always refers to a particular one of the three components. This might be easiest to achieve in practice using a two-step approach: First, obtain results for a model in which the block structure is assumed the same for all three components (as depicted in Figure 1). Then, use the final posterior probabilities of component inclusion as starting values for a second algorithm for fitting the more general model.

\section{Examples for real and simulated datasets}

\subsection{A simulation study}

We applied the nonparametric EM algorithm (npEM) to the same synthetic examples for which Hall et al. (2005) tested their estimation technique, a method based on inverting the mixture model. The three simulated models, described below, are trivariate two-component mixtures $(m=2, r=3)$ with independent repeated measures, i.e., $b_{k}=k$ for $1 \leq k \leq 3$. We ran $S=300$ replications of $n=500$ observations each and computed the errors in terms of the square root of the Mean Integrated Squared Error (MISE) for the densities as in Hall et al. (2005), where

$$
\operatorname{MISE}_{j k}=\frac{1}{S} \sum_{s=1}^{S} \int\left(\hat{f}_{j k}^{(s)}(u)-f_{j k}(u)\right)^{2} d u, \quad j=1,2 \text { and } k=1,2,3 ;
$$


and the integral is computed numerically. Each density $\hat{f}_{j k}^{(s)}$ is computed using equation (9) together with the posterior probabilities after convergence of the algorithm, i.e., the final values of the $p_{i j}^{t}$.

As suggested in section 3.1, we started each algorithm with an initial $n \times m$ matrix $\mathbf{P}^{0}=\left(p_{i j}^{0}\right)$, and this matrix was determined by a k-means algorithm applied to each trivariate dataset, with initial cluster centers $(0,0,0)$ and $(4,4,4)$. This testing protocol is adapted to this particular locationshifted model in order to prevent label-switching among replications. As explained in Section 4.4, "label-switching" refers to the fact that arbitrary re-orderings of the component labels $1, \ldots, m$ are possible when numerous instances of nearly the same mixture problem are solved. Label-switching can be problematic in simulation studies, bootstrap estimation, or MCMC implementations when mixture models are present. For more discussion of the label-switching problem, see MacLachlan and Peel (2000, section 4.9) or Celeux et al. (1996). Hall et al. (2005) dealt with label-switching in the current context by enforcing the constraint $\hat{\lambda}_{1}<\hat{\lambda}_{2}$. After finishing our simulation, we verified that our results would not have changed if we had used the Hall et al. approach because in every trial, we observed that $\hat{\lambda}_{1}<\hat{\lambda}_{2}$.

To set up tuning parameters (including bandwidth, though their inversion method has several other tuning parameters), Hall et al. (2005) used near-optimal values derived by fitting a Gaussian model. With our method, we simply used the default bandwidth described in Section 3.2.

The first example is a normal model, for which the individual densities $f_{j \ell}$ are the pdf's of $\mathcal{N}\left(\mu_{j \ell}, 1\right)$, with component means $\boldsymbol{\mu}_{1}=(0,0,0)$ and $\boldsymbol{\mu}_{2}=(3,4,5)$. The second example uses double exponential distributions with densities $f_{j \ell}(t)=\exp \left\{-\left|t-\mu_{j \ell}\right|\right\} / 2$, where $\boldsymbol{\mu}_{1}=(0,0,0)$ and $\boldsymbol{\mu}_{2}=(3,3,3)$. In the third example, the individual distributions have cen- 

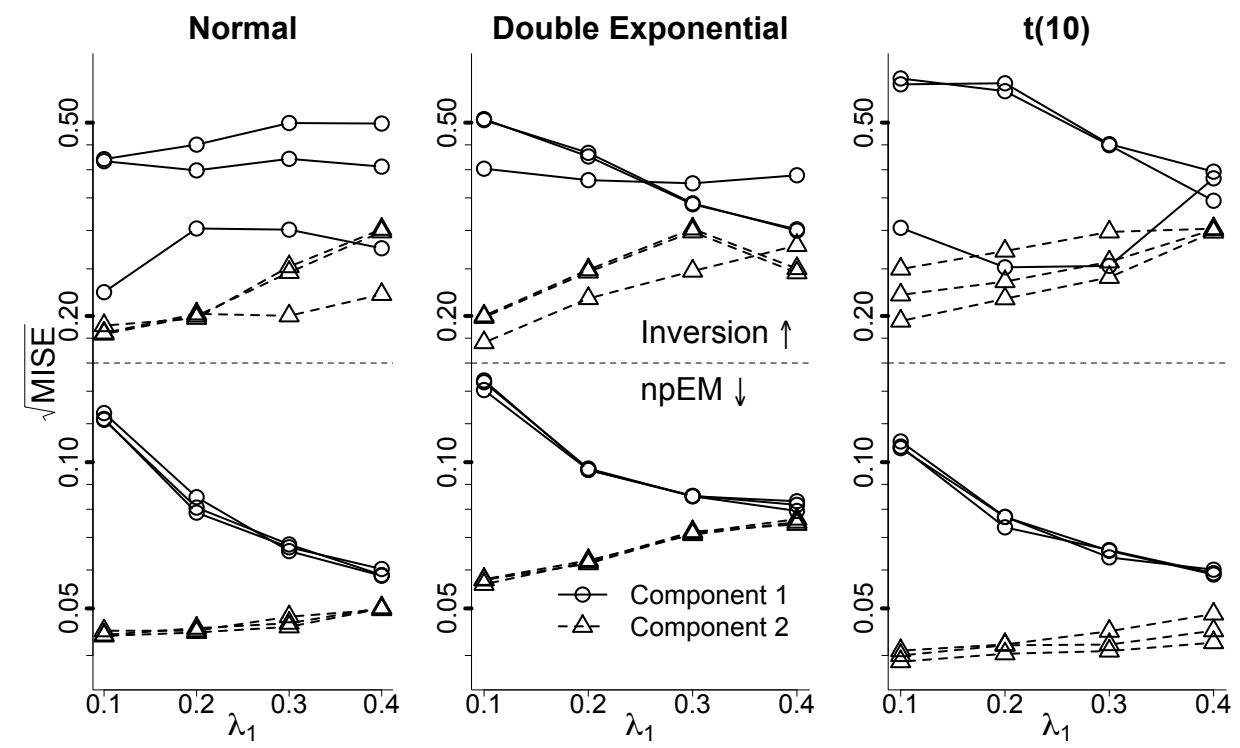

Figure 2: Square roots of Mean Integrated Squared Errors (MISE) are shown on a logarithmic scale as a function of $\lambda_{1}$, the proportion of component 1 , for three different simulated distributions for all $f_{j k}, j=1,2$ and $k=1,2,3$. The results for the inversion algorithm of Hall et al. (2005) are approximated from their plots on page 675, Fig. 2, with a small bit of noise added to separate coincident points.

tral or noncentral $t$ densities on ten degrees of freedom: The first component has a central $t(10)$ distribution and thus $\boldsymbol{\mu}_{1}=(0,0,0)$, whereas the second component's coordinates are noncentral $t(10)$ distributions with noncentrality parameters 3,4 , and 5 . Thus, the mean of the third component is $\boldsymbol{\mu}_{2}=(3,4,5) \times 1.0837$. Recall that in all three examples - and indeed throughout this article — the coordinates are independently distributed conditional on their component membership. We stress that even though the true models in the normal and double exponential examples are special cases of equation (17), the algorithm used for the simulation assumes only the general model (4) with $b_{k}=k$ for all $k$. 
Results given in Figure 2 show that our algorithm dramatically outperforms the inversion method for the three test cases. Note that the smallest value of MISE for the inversion method for any example is greater than the greatest value of MISE for our npEM algorithm; thus, when all of the results are plotted on the logarithmic axes of Figure 2, the horizontal dotted

line at $\sqrt{\mathrm{MISE}}=0.16$ separates the $\mathrm{npEM}$ results from the inversion results entirely. Because the three coordinates within each component and value of $\lambda_{1}$ are so similar relative to the scale of the plots, we do not distinguish among them in Figure 2. Predictably, we can see that the MISE is much smaller for the second component than the first when $\lambda_{1}$ is small (which means a larger proportion of the sample gives information about the second component), but the values appear to converge as $\lambda_{1}$ nears $1 / 2$.

\subsection{Other simulation studies}

In addition to the example of Hall et al. (2005), we tested our algorithm on several other simulation examples drawn from the literature on semiparametric mixture models, specifically those of Elmore (2003), Hettmansperger and Thomas (2000), and Qin and Leung (2006). Each of these examples involves two components $(m=2)$. Table 1 summarizes the three models we tested, one from each of these articles.

Both Elmore (2003) and Hettmansperger and Thomas (2000) consider mixtures of multivariate normal distributions in which each coordinate is identically distributed (i.e., the special case of Equation (4) in which $b_{k}=1$ for all k). They each apply the cutpoint approach described in Section 2, though Hettmansperger and Thomas always use just a single cutpoint whereas Elmore tries different numbers of cutpoints. In the examples with which we compare, Elmore obtains the minimum or close to the minimum 
Source; sample size, number of coordinates; number of simulations run Model for mixture density $g(\mathbf{x})$

(a) Elmore (2003); $n=100, r=8 ; 1000$ replications

$$
\lambda_{1} \prod_{k=1}^{8} \phi\left(x_{k} ; 0,1\right)+\left(1-\lambda_{1}\right) \prod_{k=1}^{8} \phi\left(x_{k} ; 1,1\right)
$$

(b) Hettmansperger and Thomas (2000); $n=20, r=6$; 5000 replications

$$
\frac{1}{3} \prod_{k=1}^{6} \phi\left(x_{k} ; 0,1\right)+\frac{2}{3} \prod_{k=1}^{6} \phi\left(x_{k} ; \mu_{2}, 1\right)
$$

(c) Qin and Leung (2006); $n=500, r=3 ; 500$ replications

$$
\lambda_{1} \prod_{k=1}^{3} \phi\left(x_{k} ; 0,1\right)+\left(1-\lambda_{1}\right) \phi\left(x_{1} ; 1,1\right) \phi\left(x_{2} ; 1.5,1\right) \phi\left(x_{3} ; 2,1\right)
$$

Table 1: Some 2-component test simulation examples from the literature, where $\phi(\cdot ; \mu, 1)$ denotes the density of the normal distribution $\mathcal{N}(\mu, 1)$ with mean $\mu$ and standard deviation 1 .

mean square error using five cutpoints, so it is the 5-cutpoint results that we report in Table 2 .

Qin and Leung (2006) consider the more general possibility that each of the coordinates has a distinct distribution (i.e., the special case of Equation (4) in which $b_{k}=k$ for all $k$ ). However, their model entails an assumption that Equation (4) does not, which is that for each coordinate $1 \leq k \leq r$, the densities $f_{1 k}(\cdot)$ and $f_{2 k}(\cdot)$ are related by the relationship

$$
f_{2 k}(t)=f_{1 k}(t)\left(\exp \left\{\alpha_{k}+t \beta_{k}+t^{2} \gamma_{k}\right\}\right)
$$

for unknown parameters $\alpha_{k}, \beta_{k}$ and $\gamma_{k}$.

We ran multiple simulations of each of the examples (the exact number is given in Table 1) using default values of the npEM function for each simulation. We started each algorithm using the true mean parameters as initial centers for the kmeans function, as described at the end of Section 3.1, instead of randomly chosen centers. We used this technique for two technical 


\begin{tabular}{|c|c|c|c|c|c|}
\hline & $\begin{array}{c}\text { Unspecified } \\
\text { parameter } \\
\text { values }\end{array}$ & $\begin{array}{c}\text { Our mean } \\
\text { bias in } \\
\text { estimating } \lambda_{1}\end{array}$ & $\begin{array}{c}\text { Our } \\
\text { MSE }\end{array}$ & $\begin{array}{c}\text { Reported } \\
\text { MSE }\end{array}$ & Ratio \\
\hline (a) & $\lambda_{1}=1 / 4$ & 0.00332 & 0.00382 & 0.00524 & 0.73 \\
(a) & $\lambda_{1}=1 / 2$ & -0.00085 & 0.00484 & 0.00247 & 1.96 \\
(b) & $\mu_{2}=1$ & 0.05458 & 0.0387 & 0.056 & 0.69 \\
(b) & $\mu_{2}=3$ & -0.00004 & 0.0109 & 0.011 & 0.99 \\
(c) & $\lambda_{1}=0.3$ & -0.00011 & 0.00083 & 0.00084 & 0.99 \\
(c) & $\lambda_{1}=0.5$ & 0.00186 & 0.00112 & 0.00098 & 1.14 \\
(c) & $\lambda_{1}=0.8$ & -0.00204 & 0.00074 & 0.00078 & 0.95 \\
\hline
\end{tabular}

Table 2: Summary of mean square errors for estimates of $\lambda_{1}$ reported by several authors for various models using their methods, along with corresponding mean bias and MSE found in our experiments. The labels (a), (b), and (c) refer to Table 1.

reasons: First, it reduced the chances of converging to a sub-optimal solution due to an unlucky choice of starting values, thus saving the trouble of using multiple starting values for each simulated dataset, as is usually prudent. Second, it provided an a priori labeling for each of the two components, which then enabled us to check to see whether any of the final solutions after convergence indicated that a component label had switched during the running of the algorithm. In our simulation studies of Sections 5.1 and 5.2, the only example in which label-switching appeared to be a problem was example (b) with $\mu_{2}=1$ from Hettmansperger and Thomas (2000), where we reversed the component labels in 57 out of 5000 cases because the final solution obtained had a larger estimated mean in the first-component density estimate than in the second-component density estimate.

Since each author reported an empirical mean square error of the $\lambda_{1}$ parameter estimate for a large number of simulations, we may compare our estimates to theirs on this basis at least. These comparisons, given in Table 2, suggest that our method produces $\lambda_{1}$ estimates of comparable 
mean square error to those of the alternative methods proposed by the various authors. Though it is difficult to generalize from such a limited test against several different estimation methods, it does appear that the great flexibility of our method does not come at great expense in terms of the MSE of parameter estimates, at least for the examples we consider here.

\subsection{The Water-level data}

The water-level dataset described in Section 1 is available in the mixtools package (Young et al., 2008) in R (R Development Core Team, 2008) by typing data("Waterdata"), and its documentation file may be accessed by then typing help("Waterdata"). These data, with $n=405$ and $r=8$, have been analyzed by other authors using nonparametric mixture models based on converting the continuous angle measurements into binomial or multinomial data (Hettmansperger and Thomas, 2000; Elmore et al., 2004). The latter of these two references gives a lengthy analysis of this dataset, which we use as a basis of comparison for our method.

By converting the water-level data into multinomial vectors, Elmore et al. (2004) are assuming that the eight coordinates of an observation vector are i.i.d. conditional on the mixture component from which the vector is drawn. Yet a more careful analysis, not possible using any previously published method we know of, reveals the differences among the coordinate distributions. Grouping the coordinates into four blocks of two i.i.d. coordinates each uses knowledge of the task (described in Section 1) and appears more appropriate here.

Figure 1 of Section 1 summarizes our three-component solution, which may be obtained using mixtools via

blockid <- $c(4,3,2,1,3,4,1,2)$ \# blocks $1-4$ refer to fig. 1 
a <- npEM(Waterdata, mu0=3, blockid=blockid, bw=4)

plot (a, hist $=\mathrm{T}$, breaks $=5 *(0: 37)-92.5)$

(type ?npEM and ?plot.npEM for more details on these functions). Note that "bw=4" specifies the bandwidth, overriding the default value of equation (10). Also note that because the default starting values are random, the commands above may not result in exactly the same solution.

For the three-component solution, Figure 1 clearly shows that the third component, comprising almost $50 \%$ of the subjects, consists of individuals who know how to complete this task; these individuals' responses are highly peaked around the correct answer of zero degrees. The cutpoint method also finds a similarly shaped component and estimates its proportion at 0.440 . However, the first and second components are qualitatively different than those found by the cutpoint method, particularly the smallest component. Using our method, we find that almost $8 \%$ of the subjects seem to draw the line parallel to the bottom of the vessel - yet the cutpoint approach misses this group because "parallel to the bottom" means one of $-60,-30,30$, or 60 degrees depending on the orientation the vessel. In fact, the assumption that all eight coordinates are identically distributed leads the cutpoint approach to conclude that the smallest component (with an estimated $17.7 \%$ of all subjects) is roughly uniformly distributed over the interval from -90 to 90 . The more realistic model that is possible to estimate with our algorithm reveals details that the cutpoint approach simply cannot find easily.

In fact, the cutpoint approach can find the group of subjects who draw the water level parallel to the bottom of the vessel, but it needs a fourcomponent model to do so. Elmore et al. (2004, Fig. 2) give a cutpoint solution for the four-component case, with $3.3 \%$ of the subjects in which the density has four sharp peaks at $-60,-30,30$, and 60 degrees. But this result 

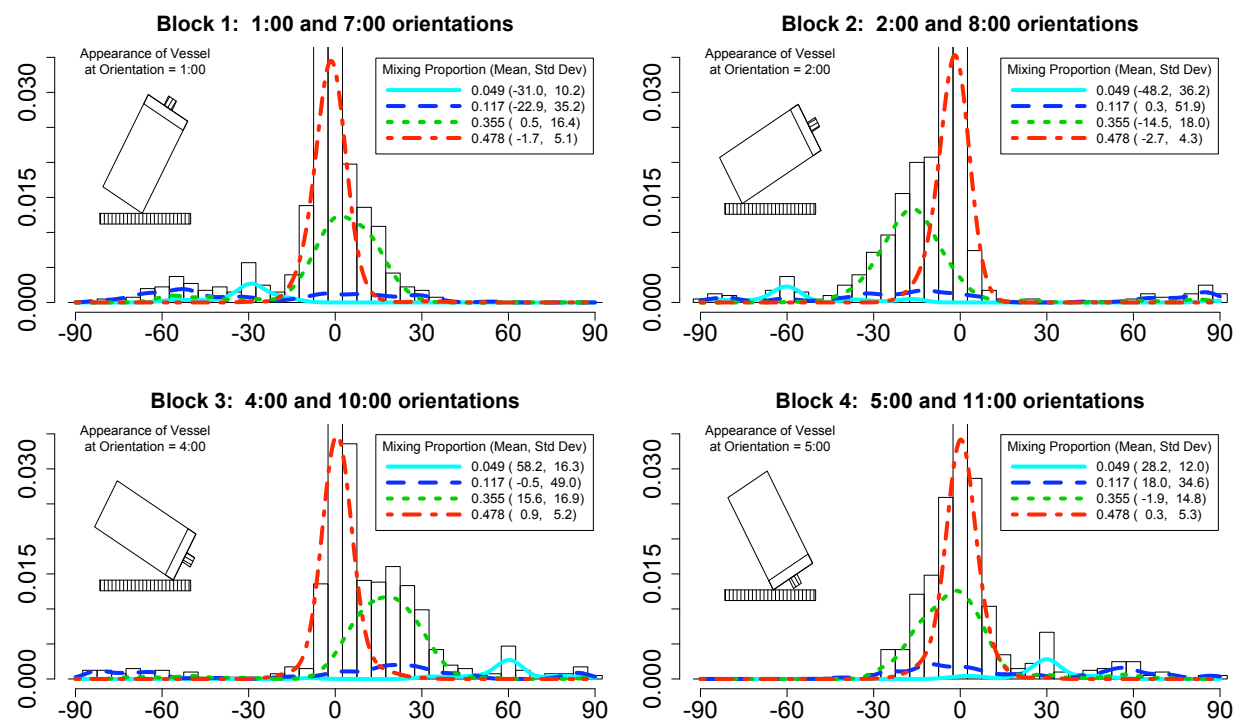

Figure 3: Results of a four-component analysis of the water-level data using our algorithm. The means and standard deviations are not part of the nonparametric model and are included for interpretation only.

masks the fact that those peaks occur in completely different coordinates, so the implicit assumption of conditionally i.i.d. coordinates using the cutpoint approach is evidently not appropriate here.

We include the analogous four-component solution using our method, obtained by simply changing the 3 to a 4 in the second line of the code given earlier, as Figure 3. We stress that the means and standard deviations reported in Figures 1 and 3 are only for aiding the interpretation of the density estimates; they are not part of the model and depend only on the final values of $p_{i j}$ and the original data. Formulas for them are identical computationally to those given for $\mu_{j l}^{t+1}$ and $\sigma_{j l}^{t+1}$ in equations (13) and (14), each of which also relies on the formula for $\lambda_{j}^{t+1}$ in equation (7).

It is possible to obtain confidence intervals on the Euclidean parameter 
estimates (the $\lambda_{j}$ ) using a nonparametric bootstrap approach by repeatedly resampling with replacement from the empirical distribution defined by the $n$ observed $r$-dimensional vectors. Though a complete treatment of this topic is beyond the scope of this article, we will simply mention that bootstrapping in the context of mixture models can be challenging because of the labelswitching problem referred to in section 5.1. In the three-component waterlevel dataset analysis, we obtained empirical 95\% confidence intervals for $\lambda_{1}$, $\lambda_{2}$, and $\lambda_{3}$ of $(0.054,0.152),(0.338,0.467)$, and $(0.446,0.552)$, respectively using 10,000 bootstrap samples. Furthermore, we found no evidence of labelswitching, which is unsurprising because the three components are quite easily distinguished from one another.

As stated in Section 2, a word of caution is necessary here: it has not been proven that the general model (4) is identifiable when $r=8$ and $m=3$ or 4. By contrast, under the more restrictive assumptions of the cutpoint method, we know that identifiability holds in these cases because $r \geq 2 m-1$ (Elmore and Wang, 2003). The necessary (but not sufficient) lower bounds on $r$ given by Hall et al. (2005) are $r \geq 4$ when $m=3$, and $r \geq 5$ when $m=4$; so with $r=8$ there is at least the hope of identifiability in each case. We are encouraged in the present example by several facts: First, the solutions we obtain, for both $m=3$ and $m=4$, are stable in the sense that we obtain the same solutions repeatedly for different randomly selected starting values for the algorithm. Furthermore, our results may be explained qualitatively via an understanding of how the data arose, and these results confirm and sharpen those found using a different method, the cutpoint method, in which identifiability is known to hold. 


\subsection{Stochastic vs. non-stochastic semiparametric EM}

For Model (19) with $m=2$ components, we compare the semiparametric stochastic EM (spSEM) algorithm of Bordes et al. (2007), which is discussed immediately following equation (9), with the deterministic semiparametric EM (spEM) algorithm that uses equation (20).

\begin{tabular}{|c|ccc|ccc|}
\hline & $\lambda$ & $\mu_{1}$ & $\mu_{2}$ & $\lambda$ & $\mu_{1}$ & $\mu_{2}$ \\
True & 0.25 & -1 & 2 & 0.25 & -1 & 2 \\
\hline \hline & \multicolumn{3}{|c|}{ MSE } & \multicolumn{3}{c|}{ bias } \\
\hline spSEM & 0.0044 & 0.1880 & 0.0459 & -0.0246 & 0.0413 & -0.1003 \\
spEM & 0.0042 & 0.1154 & 0.0373 & -0.0229 & 0.0056 & -0.0898 \\
\hline \hline
\end{tabular}

Table 3: Empirical mean squared error (MSE) and bias for $\left(\lambda, \mu_{1}, \mu_{2}\right)$, based on 10, 000 Monte-Carlo replications of Model (19) with $f(\cdot)$ taken to be standard normal and $n=100$. The spSEM and spEM algorithms are run for 100 and 20 iterations each, respectively, starting from the true parameter values.

The comparison is based on 10,000 Monte Carlo replications in which we selected the bandwidth $h$ according to the formula used by Bordes et al. (2007), namely, $h=1.06 n^{-1 / 5}$, or $h=0.422$ when $n=100$. [NB: This formula is given by Scott (1992) and is different from the default value of equation (10), in which 1.06 is replaced by 0.9.] The spEM was allowed only 20 iterations, instead of the 100 iterations allowed the stochastic version, since its non-stochastic sequence of estimates requires fewer iterations to stabilize. Results are given in Table 3 and give empirical evidence that the deterministic version is slightly more efficient than the stochastic version. 


\subsection{Empirical convergence rates}

Finally, we include a simulation study whose purpose is to explore the possible rate of convergence for the algorithm, for fixed $m$ and $r$, as the sample size $n$ tends to infinity. Note that the plots here do not constitute a proof of the asymptotic rate of convergence, nor even of consistency, yet they are interesting nonetheless because they suggest that such a theoretical result is possible.
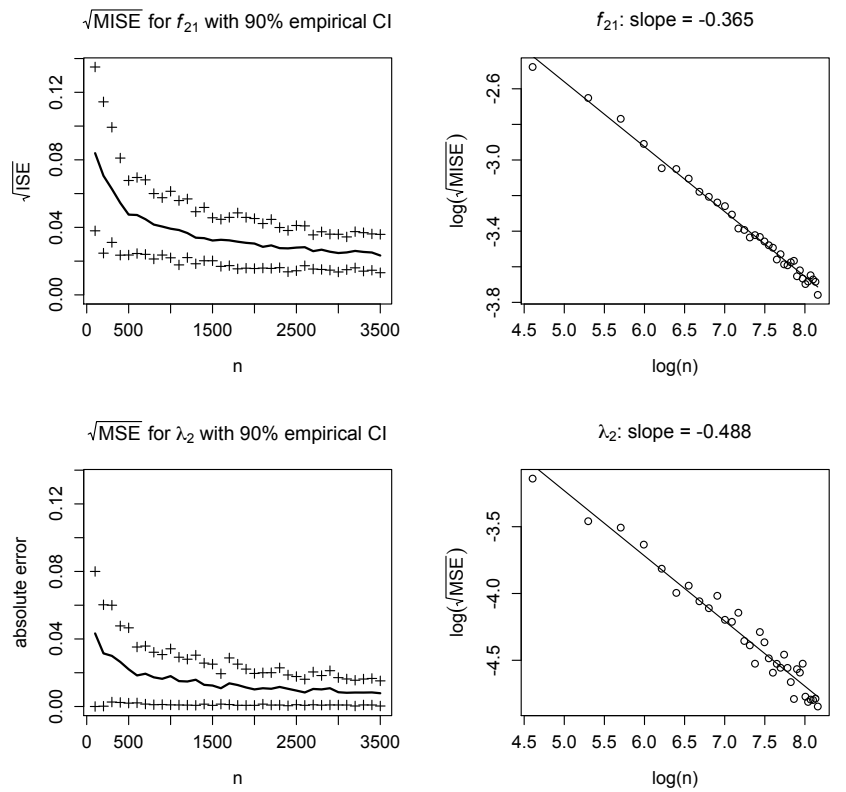

Figure 4: Errors as a function of sample size for one of the six densities, $f_{21}$, and its corresponding proportion $\lambda_{2}$. On the left are the root-mean errors along with $90 \%$ empirical confidence intervals; on the right are log-log plots of the root-mean errors along with least-squares fits.

Figure 4 gives results for a simulation using the normal example of Section 5.1 with $m=2$ components and $r=3$ independent (but not identically distributed) coordinates, for which the individual densities $f_{j \ell}$ 
are from $\mathcal{N}\left(\mu_{j \ell}, 1\right)$ with $\lambda_{1}=0.4$ and component means $\boldsymbol{\mu}_{1}=(0,0,0)$ and $\boldsymbol{\mu}_{2}=(3,4,5)$. We ran 100 replications for each of the sample sizes $n=100,200, \ldots, 3500$. Bandwidths are chosen using the default method of Section 3.2. Only one of the six $f_{j \ell}$ densities is shown in Figure 4, but the other five plots are nearly identical in appearance and empirical rate. The empirical rate of -0.488 for the Euclidean parameter $\lambda_{2}$ is close to the optimal rate of $n^{-1 / 2}$ for the usual parametric case. The rate of -0.365 for the density estimate (the other five rates range from -0.361 to -0.370 ) is somewhat below the optimal rate of $n^{-2 / 5}$ for a standard kernel density estimate. Yet as we explain in Section 3.2, the bandwidth is probably not optimal in any sense, and indeed the density estimation problem in the mixture setting may well be a more difficult problem than in the non-mixture setting.

The empirical rate of convergence for the Euclidean parameter suggests that asymptotic normality may be expected. We thus also provide the empirical distribution of $\lambda_{1}$ from a sample $\left(\hat{\lambda}_{1}^{(s)}, s=1, \ldots S\right)$ of estimates of $\lambda_{1}$ based on $S=300$ replications (as in Section 5.1). This distribution depicted in Figure 5 looks reasonably normal, and a Shapiro-Wilk test for normality gives a $p$-value of 0.87

\section{Discussion}

The algorithm we propose in this article is the first algorithm we have seen in the literature for dealing with model (4) in its full generality. Furthermore, it is quite a bit easier to code than many if not all competing algorithms in the particular cases to which the latter are suited. Finally, we have given empirical evidence that our algorithm produces dramatically lower error rates than the inversion method of Hall et al. (2005) for the test cases used 


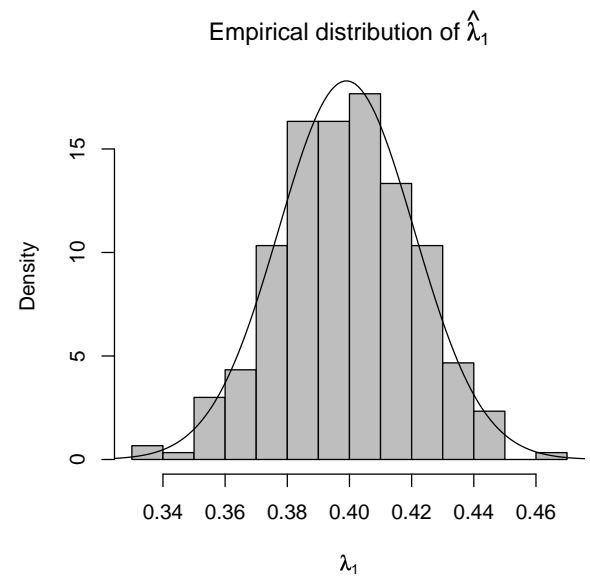

Figure 5: Empirical distribution of $\hat{\lambda}_{1}$ for the gaussian model with $\lambda_{1}=0.4$, based on 300 replications of samples of size $n=500$.

in that paper, and we have explained how our algorithm gives insight into the multivariate mixture structure of a particular dataset (the water-level dataset) that is not possible under the more restrictive assumption that each multivariate observation has conditionally i.i.d. coordinates.

As we point out in Section 2, the great flexibility of our method requires some caution, since it is very easy to apply the algorithm for arbitrary $m$ (number of mixture components) and $r$ (number of vector coordinates per observation) even when model (4) is not known to be identifiable. We know that model (4) is not identifiable for an arbitrary $m$ and $r$; yet it is not yet known where the "identifiability boundary" might lie — i.e., for which values $\rho(m)$ it is true that $r \geq \rho(m)$ implies identifiability but $r \geq \rho(m)-1$ does not. Hall and Zhou (2003) proved that $\rho(2)=3$ as long as a weak condition they term "irreducibility" is satisfied, and Hall et al. (2005) and Elmore et al. (2005) have made some progress towards a general solution, but so far such a solution remains elusive. Our empirical experience with 
our algorithm suggests that it is very easy to find examples when $r=1$ and $m=2$ in which nonidentifiability clearly leads to spurious estimates; yet for $r=2$ and $m=2$, it is not easy to construct such examples. In fact, in this case of two components and two coordinates, Hall and Zhou (2003) argue that the model is "almost" identifiable, a concept they make precise in their article.

There are several questions about our algorithm that could be further investigated in addition to the identifiability question. For instance, Hall et al. (2005) introduce a further generalization of model (4). Namely, they allow some of the $f_{j \ell}(\cdot)$ to be multivariate densities whose coordinates are not independent. There is no difficulty in extending our algorithm to this case in principle, though to do so requires the use of multidimensional kernel density estimates. We have not explored this possibility yet. Furthermore, some of the questions raised by Bordes et al. (2007) in the context of the stochastic EM-like algorithm they developed for the univariate case also apply to our algorithm, since the two algorithms are quite similar in principle. For example, it might be that there is an analogue to their Lemma 1, which proves a sort of "fixed-point" property: In our case, it would be reasonable to ask whether our algorithm would stay near the true value at the next iteration if started exactly at the truth. Furthermore, there is the question of which, if any, function is being maximized by our algorithm and whether each iteration leads to an increase in this function (as is the case for a true EM algorithm, which increases the true loglikelihood function at each iteration).

Selection of an appropriate bandwidth is another area in which further work could shed some light. We have discussed this problem at length in Section 3.2. Indeed, selecting a bandwidth in a mixture setting like this one 
appears to be a fundamentally more complicated problem than the corresponding non-mixture case due to the fact that we do not have a sample from any of the individual mixture components per se, and we do not obtain information on the individual components until after the algorithm has already been run. This suggests an iterative scheme as mentioned in Section 3.2 , but we do not test such a scheme in the current article. Related to the bandwidth selection question is the question of whether our algorithm can be shown to be consistent for a fixed $r$ and $m$; and if so, at what rate it converges. Preliminary empirical evidence, discussed in Section 5.5, suggests that this rate of convergence is comparable to usual rates for the Euclidean parameters and perhaps slightly slower than usual rates for the kernel density estimates.

Anecdotally, the convergence properties of our algorithm appear to be much like those of a typical EM algorithm. For one thing, our algorithm sometimes attains different solutions when given different starting parameters, which is analogous to an EM algorithm's local, rather than global, maximization properties (though as we point out above, the question of whether our algorithm actually maximizes some function is as yet unanswered). Furthermore, as with any mixture problem, care must be exercised to avoid label-switching problems in situations where it is important that the component labels of the estimates match some a priori pattern; and this caveat is particularly true when components are not well-separated.

Finally, we reiterate that the analyses in this article may be reproduced using the publicly-available $\mathrm{R}$ package called mixtools ( $\mathrm{R}$ Development Core Team, 2008; Young et al., 2008). Future revisions of this package may extend its capabilities to include some of the discussion items here.

\section{Acknowledgment}


The authors are grateful to the reviewers for their constructive comments and helpful suggestions. This research is partially supported by NSF Award SES-0518772. DRH wishes to thank Le Studium, CNRS Orléans, France for additional support of this research.

\section{References}

Anderson, J. A. (1979), Multivariate logistic compounds, Biometrika, 66: $17-26$.

Bordes, L., Mottelet, S., and Vandekerkhove, P. (2006), Semiparametric estimation of a two-component mixture model, Annals of Statistics, 34, $1204-1232$.

Bordes, L., Chauveau, D., and Vandekerkhove, P. (2007), An EM algorithm for a semiparametric mixture model, Computational Statistics and Data Analysis, 51: 5429-5443.

Celeux, G., Chauveau, D., and Diebolt, J. (1996), Stochastic versions of the EM algorithm: An Experimental Study in the Mixture Case, Journal of Statistical Computation and Simulation, 55, 287-314.

Cruz-Medina, I. R., Hettmansperger, T. P. and Thomas, H. (2004), Semiparametric mixture models and repeated measures: the multinomial cut point model, Journal of the Royal Statistical Society, Series C, 53: 463474 .

Dempster, A., Laird, N. and Rubin, D. (1977). Maximum likelihood from incomplete data via the EM algorithm (with discussion). J. Roy. Stat. Soc., B, 39, 1-38.

Elmore, R. T. (2003), Semiparametric Analysis of Finite Mixture Models with Repeated Measures, Ph.D. thesis, the Pennsylvania State University.

Elmore, R. T. and Wang, S. (2003), Identifiability and estimation in finite mixture models with multinomial coefficients, Penn State Department of Statistics Technical Report \#03-04.

Elmore, R. T., Hettmansperger, T. P., and Thomas, H. (2004), Estimating component cumulative distribution functions in finite mixture models, Communications in Statistics: Theory and Methods, 33: 2075-2086.

Elmore, R. T., Hall, P. and Neeman, A. (2005), An application of classical invariant theory to identifiability in nonparametric mixtures, Annales de l'Institut Fourier, 55, 1: 1-28. 
Hall, P. and Zhou, X. H. (2003) Nonparametric estimation of component distributions in a multivariate mixture, Annals of Statistics, 31: 201224 .

Hall, P., Neeman, A., Pakyari, R., and Elmore, R. (2005), Nonparametric inference in multivariate mixtures, Biometrika, 92: 667-678.

Hettmansperger, T. P. and Thomas, H. (2000), Almost nonparametric inference for repeated measures in mixture models, Journal of the Royal Statistical Society, Series B, 62, 811-825.

Hunter, D. R., Wang, S., and Hettmansperger, T. P. (2007), Inference for mixtures of symmetric distributions, Annals of Statistics, 35: 224-251.

Leung, D. H.-Y. and Qin, J. (2006), Semi-parametric inference in a bivariate (multivariate) mixture model, Statistica Sinica, 16: 153-163.

Lindsay, B. G. (1995) Mixture Models: Theory, Geometry and Applications, Hayward, CA: Institute of Mathematical Statistics.

McLachlan, G. and Peel, D. A. (2000) Finite Mixture Models, New York: Wiley.

Qin, J. and Leung, D. H.-Y. (2006), Semiparametric analysis in conditionally independent multivariate mixture models, unpublished manuscript.

R Development Core Team (2008). R: A language and environment for statistical computing. R Foundation for Statistical Computing, Vienna, Austria. ISBN 3-900051-07-0, URL http://www.R-project.org.

Scott, D. W. (1992), Multivariate Density Estimation: Theory, Practice, and Visualization, Wiley.

Silverman, B. W. (1986), Density Estimation, London: Chapman and Hall.

Thomas, H., Lohaus, A., and Brainerd, C.J. (1993). Modeling Growth and Individual Differences in Spatial Tasks, Monographs of the Society for Research in Child Development, 58, 9: 1-190.

Titterington, D. M., Smith, A. F. M., and Makov, U. E. (1985). Statistical Analysis of Finite Mixture Distributions, Chichester, Great Britain: Wiley.

Young, D. S., Benaglia, T., Chauveau, D., Elmore, R. T., Hettmansperger, T. P., Hunter, D. R., Thomas, H., and Xuan, F. (2008) mixtools: Tools for mixture models, $\mathrm{R}$ package version 0.3.2. 